Tributyltin is a potent inhibitor of piscine peroxisome proliferator-activated receptor $\alpha$ and $\beta$

Louise Colliar, Armin Sturm and Michael J. Leaver

Comparative Biochemistry and Physiology Part C: Toxicology \& Pharmacology Volume 153, Issue 1, January 2011, Pages 168-173

http://dx.doi.org/10.1016/j.cbpc.2010.10.008

Author's final refereed version: http://hdl.handle.net/1893/2832

NOTICE: this is the author's version of a work that was accepted for publication in Computer Networks. Changes resulting from the publishing process, such as peer review, editing, corrections, structural formatting, and other quality control mechanisms may not be reflected in this document. Changes may have been made to this work since it was submitted for publication. A definitive version was subsequently published in Comparative Biochemistry and Physiology Part C: Toxicology \& Pharmacology, 153, 1, (2011) j.cbpc.2010.10.008 


\title{
Tributyltin is a potent inhibitor of piscine peroxisome proliferator-activated receptor $\alpha$ and $\beta$
}

Louise Colliar $^{\mathrm{a}}$, Armin Sturm ${ }^{\mathrm{a}}$, Michael J. Leaver ${ }^{\mathrm{ab}}$.

a, Institute of Aquaculture, University of Stirling, Stirling, FK9 4LA, United Kingdom

b, corresponding author: email mj11@stir.ac.uk

$$
\begin{aligned}
& \text { tel }+44(0) 1786467995 \\
& \text { fax }+44(0) 1786476133
\end{aligned}
$$

\begin{abstract}
Abbreviations: TBTO, bis [tri-n-butyltin] oxide); PPAR, peroxisome proliferator-activated receptor; RXR, 9-cis retinoic acid receptor; LBD, ligand binding region; DTT, dithiothreitol; DMEM, Dulbeccos modified Eagles medium; OEA, N-oleoyl ethanolamine; LEA, N-Linoleyl ethanolamine; DHA, docosahexaenoic acid
\end{abstract}

\begin{abstract}
Increasing evidence suggests that common environmental contaminants can act as endocrine disrupters in fish. However, current data are biased towards environmental estrogens, highlighting the need to elucidate potential pollutant impact on other endocrine axes. Here, we report a highthroughput assay to identify chemicals interacting with piscine peroxisome proliferator-activated receptors (PPARs). Our transactivation assay employs a fish cell line and uses recombinant proteins combining the yeast Gal4 DNA-binding domain with the ligand-binding domain of PPARs from plaice. Compared to assays with full-length PPARs, this approach circumvents interaction of chemicals binding to retinoid $\mathrm{X}$ receptors, which form heterodimers with PPAR and many other nuclear receptors. Plaice PPAR $\alpha$ and PPAR $\beta$ are activated by fibrate drugs and by phthalate monoesters at concentrations similar to those activating the homologous mammalian receptors. In line with their assumed role as central transcriptional regulators of energy homostasis, a number of fatty acids activate plaice PPAR $\alpha$ and PPAR $\beta$. In contrast, tributyl tin oxide (TBTO) is a potent antagonist of PPAR $\alpha$ and PPAR $\beta$, showing activity at environmentally relevant concentrations of TBTO (1-50 nM). Given the ubiquitous and persistent nature of TBTO, the possibility that chronic environmental effects are occurring via disruption of PPAR signalling in fish should be further investigated.
\end{abstract}

Keywords: tributyltin, TBTO, PPAR, pollutant, fibrate, phthalate, endocrine disruptor 


\section{Introduction}

PPARs have critical roles in regulating energy metabolism and particularly lipid utilization and storage (Feige et al., 2006). PPARs are members of the nuclear hormone receptor (NHR) family and are encoded by three genes in mammals, PPAR $\alpha$ (NR1C1), PPAR $\beta$ (NR1C2) and PPAR $\gamma(\mathrm{NR} 1 \mathrm{C} 3)$. PPARs act as heterodimers with retinoid X receptors (RXRs) and both RXR agonists and PPAR agonists activate transcription of PPAR target genes (Desvergne et al., 2006). Reflecting their roles in lipid homeostasis, natural endogenous activating ligands for PPARs include fatty acids and fatty acid metabolites and targets include a broad range of genes involved in lipid and carbohydrate metabolism (Hihi et al., 2002). The role of PPAR $\alpha$ is hypothesised to be primarily in controlling the reversible induction of $\beta$-oxidation in specific tissues, especially liver, as a response to changing energy requirements and nutritional status. The evidence for this comes most directly from rodents where PPAR $\alpha$ is expressed in cells with high catabolic rates of fatty acid oxidation such as hepatocytes, cardiomyocytes, kidney proximal tubules and intestinal mucosa (Escher et al., 2001). PPAR $\alpha$-null mice are incapable of up-regulating fatty acid oxidation during fasting (Kersten et al., 1999; Leone et al, 1999). In contrast, mammalian PPAR $\gamma$ is considered to play a critical role in fat accumulation particularly in adipocytes, and in lipid-accumulating macrophages (Rosen and Speigelman, 2001). The role of PPAR $\beta$ is less well understood, although various studies suggest that PPAR $\beta$ has a role in global control of lipid homeostasis in mammals (Wagner and Wagner, 2010). Several synthetic PPAR agonists have been discovered and some are in use as drug treatments for dyslipidemia associated with coronary heart disease and diabetes (Fievet and Staels, 2009).

PPARs can also be activated by binding environmental pollutants. For example, TBTO, the main chemical constituent of TBT-based marine antifouling paints, has been shown to bind and activate mammalian PPAR $\gamma$ at nM concentrations (Grün et al., 2006;Hiromori et al., 2009) and PPAR $\alpha$ is activated by phthalate monoesters (Bility et al., 2004). The possibility that environmental contaminants interact with human PPARs has led to the hypothesis that some of these chemicals could disrupt lipid and energy homeostasis, and might thus be a factor in the increasing prevalence of some chronic conditions such as diabetes and obesity (Grün and Blumberg, 2009). In addition, there is increasing concern that synthetic PPAR ligands used as pharmaceuticals are entering the environment in significant quantity and consequently adversely affecting wildlife (Rosal et al., 2010).

Whilst, PPAR-dependent effects are suggested to occur in humans exposed to environmental 
pollutants or pharmaceuticals, little is known about the potential effects of such chemicals on PPARs from other vertebrates. Fish species may be particularly susceptible to PPAR disruption since they can be continuously exposed in waters which receive effluents and they have similar genes and endocrine pathways to mammals. PPARs have been characterized in various fish species, and it is apparent that fish possess homologues of mammalian PPAR $\alpha, \operatorname{PPAR} \beta$ and PPAR $\gamma$ (Leaver et al., 2005; Leaver et al., 2007).

The aim of this study was to assess the effectiveness of several common environmental pollutants and discharged pharmaceuticals to activate or inhibit fish PPARs. To achieve this, and to exclude RXR mediated effects, we transfected a fish cell line with plaice PPAR $\alpha$, PPAR $\beta$ and PPAR $\gamma$ ligand-binding domains fused to the DNA binding domain of the yeast transcription factor Gal4. The results show that, in response to model PPAR ligands, the products of these constructs strongly activate a Gal4-driven luciferase reporter gene in co-tranfected fish cells. In comparison, environmental pollutants such as the fibrate drugs and monophthlate esters are weak activators of fish PPAR $\alpha$ and PPAR $\beta$, whilst TBTO strongly antagonises the activity of plaice PPAR $\alpha$ and PPAR $\beta$ at $\mathrm{nM}$ concentrations.

\section{Materials and Methods}

\subsection{Gal4-PPAR expression constructs}

Plaice PPAR $\alpha, \beta$ and $\gamma$, have been described previously (Genbank accession nos; $\underline{\mathbf{A J 5 3 9 4 6 7 . 1}}$, $\underline{\mathbf{A J 5 3 6 4 6 8 . 1}}$ and $\underline{\mathbf{A J 2 4 3 9 5 6 . 2}}$, respectively (Leaver et al., 2005)). To obtain recombinant fusion proteins in which the Gal4 DNA-binding domain was fused N-terminally to the ligand binding domain (LBD) of PPAR proteins (amino acids 153 to 475 for PPAR $\alpha, 207$ to 511 for PPAR $\beta$ and 196 to 533 for PPAR $\gamma$ ), cDNAs encoding the LBD of the individual receptoirs were amplified by PCR, using a proof-reading DNA polymerase (PfuUltraTMFusion IIHS, Stratagene). At the 5 'end of PCR primers, restriction enzyme recognition sites were added to allow the in-frame subcloning of PCR products between the BamHI and KpnI sites of the vector pBIND (Promega), using appropriate enzymes.

\subsection{Transactivation assay}

Fathead minnow (FHM) cells were maintained at $22^{\circ} \mathrm{C}$ in an atmosphere of $4 \%$ carbon dioxide in DMEM containing $4.5 \mathrm{gL}^{-1}$ glucose and $110 \mathrm{mgL}^{-1}$ sodium pyruvate and supplemented with 10 $\%$ foetal bovine serum (FBS) (Gibco), $50 \mathrm{UmL}^{-1}$ penicillin and $50 \mu \mathrm{gmL}^{-1}$ streptomycin. Twenty 
four hours prior to transfection $2 \times 10^{5}$ cells were seeded per well of a 96 well black sided, clear bottom microtitre plate (Corning). From this point on and throughout the experiments cells were maintained in DMEM as above except that directly prior to transfection FBS was replaced with $10 \%$ charcoal/dextran treated, denatured FBS (Perbio).

In transactivation assays with transiently co-transfected FHM cells, the ligand-dependent transcriptional activity of the Gal4-PPAR constructs is assessed with the help of a reporter plasmid construct in which the firefly luciferase gene is under the control of a promoter containing UAS (upstream activation sequences), which are recognised by Gal4. To control for differences in transfection efficiency, a constitutively expressed control reporter construct encoding Renilla luciferase was included in transfections. Experimental controls included treatments in which the Gal4-PPAR construct was replaced by an empty vector during transfection, as well as cells transfected with the apropriate Gal4-PPAR construct and reporters and treated with ethanol carrier only. Transfection mixtures contained, per well of a 96-well microtitre plate, $25 \mathrm{ng}$ Gal4-PPAR construct (empty vector pBIND in controls), $25 \mathrm{ng}$ Gal4UAS/luc2P-reporter plasmid (pGL4.31, Promega), $10 \mathrm{ng}$ hRluc/CMV internal control reporter plasmid (pGL4.75, Promega) and $1.2 \mu \mathrm{g}$ Polyfect transfection reagent (Qiagen), in 80 $\mu$ l of DMEM. Within each experiment, each treatment was performed in triplicate. After $24 \mathrm{~h}$, transfection mixes were removed and replaced with media containing compounds of interest. Compounds were diluted into DMEM from ethanolic stock solutions ( $5 \mu \mathrm{l}$ per $\mathrm{ml}$ ), and $75 \mu \mathrm{l}$ of the dilution was added per well. All fatty acids, pollutants and synthetic agonists were obtained from Sigma. Control treatments received ethanol only. Cells were incubated for $24 \mathrm{~h}$ before assay. Prior to luciferase measurements, media was removed and the cells washed twice with $1 \times$ PBS. Then cells were lysed by 10 min of incubation with $75 \mu 1$ per well of $1 \times$ passive lysis buffer (Promega). Firefly and Renilla luciferase activities were quantified using an assay modified from Dyer et al (2000). The firefly luciferase assay solution included 16mM DTT, $2 \mathrm{mM}$ magnesium sulphate, $25 \mathrm{mM}$ Tris/phosphoric acid (pH 8.0), 1mM EDTA, 0.5mM ATP

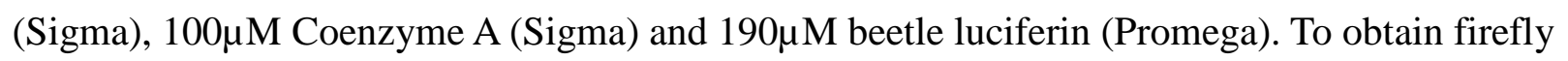
luciferase activities, $75 \mu 1$ of firefly luciferase assay solution was added per well containing $75 \mu 1$ of cell lysate, and the plate read in a Wallac Victor2 1420 luminometre. To obtain Renilla luciferase activities, $75 \mu \mathrm{l}$ per well of Renilla luciferase buffer $(0.5 \mathrm{M}$ sodium chloride, $100 \mathrm{mM}$ sodium phosphate (pH 5.1), 4mM EDTA, $0.33 \mathrm{mgmL}^{-1}$ BSA (Sigma), $0.67 \mathrm{mM}$ sodium azide and $1 \mu \mathrm{M}$ coelentrazine) was added and the plate read again. We had previously established in optimisation experiments that firefly luciferase activities were completely quenched upon addition of Renilla luciferase buffer, and that both luciferase light outputs remained constant for up to 30 mins in each of the assay conditions.

To obtain transctivation activities, firefly luciferase activities were divided by Renilla luciferase 
activities to normalise for transfection variability. Data are either shown as raw transactivation activities, or expressed as fold-induction compared to the ethanol treatment of the appropriate Gal4PPAR construct (induction experiments), or, in antagonist experiments, as the percentage reduction in activity compared to activation by PPAR model inducers Wy14643 or GW501516. Data shown are the means of at least three independent experiments.

The effects of treatments on transactivation activity were tested by one-way analyses of variance (ANOVA), followed by multiple comparisons using Dunnets post hoc test. In some cases, data were log-transformed to increase the homogeneity of variances (tested using F-max test). Where appropriate, repeated measures ANOVA was used, matching results of the same transfection. With all tests, results were considered significant when the probability value $(P)$ was $<0.05$.

\section{Results}

\section{1 $\quad$ Activation of plaice Gal4LBD constructs with PPAR agonists}

Native full-length PPAR $\alpha$ from plaice (P. platessa) has previously been shown to increase transcription of a reporter gene in a transient transfection assay following activation of the receptor by the PPAR $\alpha$-specific synthetic ligand, Wy14643 (Leaver et al., 2005). Similarly Atlantic salmon (Salmo salar) PPAR $\beta$ is activated by GW501516, a synthetic ligand developed for its specificity for mammalian PPAR $\beta$ (Leaver et al., 2007). FHM cells transfected with plaice Gal4-PPAR-LBD constructs (hereafter referred to as Gal4-PPAR $\alpha$, Gal4-PPAR $\beta$ and Gal4-PPAR $\gamma$ ) and treated with these compounds confirmed that the constructs behaved similarly to full-length PPAR receptors in terms of activation (Figure 1). Wy14643, induced luciferase luminescence 450-fold with Gal4PPAR $\alpha$, but had no effect on Gal4-PPAR $\beta$. GW501516 was an effective activator of the Gal4PPAR $\beta$ and also of Gal4-PPAR $\alpha$ constructs, with inductions of over 460 -fold and 253-fold, respectively. Bromopalmitate has previously been shown to be an activator of PPAR $\beta$ in Atlantic salmon (Leaver et al., 2007) and bromopalmitate induced transactivation of Gal4-PPAR $\beta$ and Gal4PPAR $\alpha$ over 400-fold. However, there was no response in cells transfected with the Gal4-PPAR $\gamma$ construct and treated with the mammalian PPAR $\gamma$-specific ligand rosiglitazone, or with any of the other synthetic compounds.

\subsection{Plaice PPAR $\alpha$ and PPAR $\beta$ are activated by fatty acids}

Previously, native PPAR $\alpha$ and PPAR $\beta$ from both sea bream (Sparus aurata) and plaice have been shown to respond to fatty acids, suggesting these compounds to be potential endogenous 


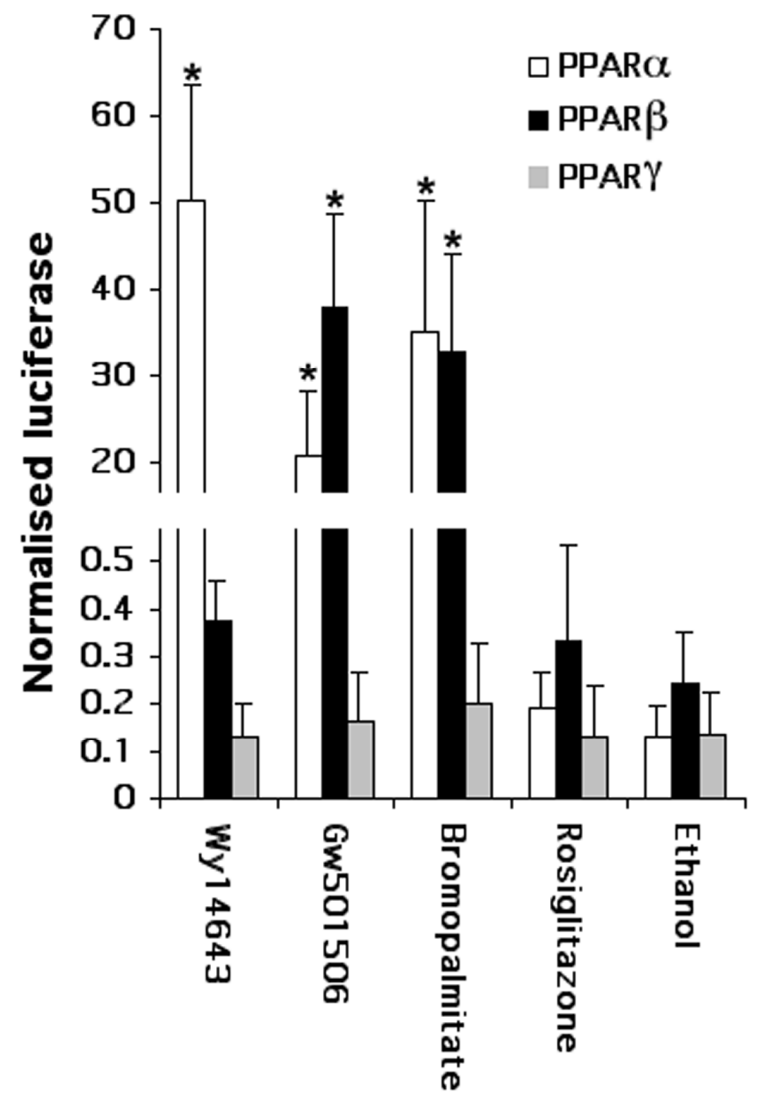

Fig. 1. Transactivation activity of Gal4-PPAR ligandbinding domain constructs in response to PPAR activators.

FHM cells were transiently transfected with Gal4-PPAR constructs, firefly luciferase reporter plasmid pGL4.31 and an internal Renilla luciferase reporter used to correct for transfection efficencies ( pGL4.75). After transfection, cells were treated (24 h) with $25 \mu \mathrm{M}$ Wy14643, $10 \mu \mathrm{M}$ GW501516, $25 \mu \mathrm{M}$ Bromopalmitate, $10 \mu \mathrm{M}$ Rosiglitazone or $0.5 \%$ ethanol vehicle. Results are expressed as arbitray units of firefly luciferase normalised to Renilla luciferase. Data represent the mean \pm SEM from three independent experiments. Asterisks indicate whether activities significantly differed from those in ethanol-treated cells $(P<0.01)$.

activators of piscine PPARs (Leaver et al., 2005). In FHM cells transfected with the Gal4PPAR $\alpha$ construct all of the tested fatty acids, with the exception of linoleic acid (18:2n-6), signficantly induced reporter gene expression. In cells transfected with Gal4-PPAR $\beta$ (Figure 2) there was was no response after oleic (18:1n-9), linoleic (18:2n-6), linolenic (18:3n-3), arachidonic (20:4n-6) or docosahexaenoic acid (22:6n-3) treatments, whereas strong responses were observed with palmitoleic, $\gamma$-linoleic ( $\gamma 18: 2)$, stearidonic (18:4n-3) and eicosapentenoic acid (20:5n-3). Noleoyl ethanolamine (OEA) and N-linoleyl ethanolamine (LEA) were effective agonists of both PPAR $\alpha$ and PPAR $\beta$. None of the fatty acids tested provoked a response with Gal4-PPAR $\gamma$ (data not shown).

\subsection{Plaice PPARs are activated by human pharmaceuticals and phthalate monoesters}

Several pollutants found in the aquatic environments were tested for potential interaction with plaice PPARs in the assay with FHM cells transfected with Gal4-PPAR $\alpha$, Gal4-PPAR $\beta$, or Gal4PPAR $\gamma$ constructs. Tested compounds included TBTO, benzylbutylphthalate and dimethylphthalate, and their mono ester metabolites, and several pharmaceutical fibrates.

The fibrates are a class of pharmaceutical compounds targeting human PPAR $\alpha$ and are used to correct lipid imbalances. Five of these compounds (gemfibrozil, bezafibrate, ciprofibrate, 


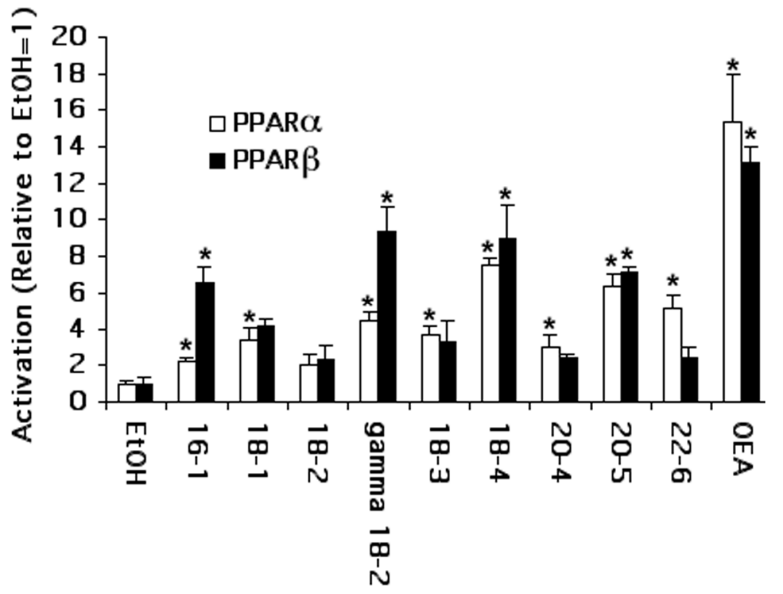

Fig. 2. Transactivation of PPAR $\alpha$ and PPARß by fatty acids and derivatives.

FHM cells were transfected and treated as described in the legend of Figure 1. Treatments included $100 \mu \mathrm{M}$ palmitoleic acid (16-1), oleic acid (18-1), linoleic acid (18-2), gamma linoleic acid ( $\gamma 18-2)$, linolenic acid (18-3), stearidonic acid (18-4), arachidonic acid (20-4), eicosapentenoic acid (20-5), docosahexaenoic acid (22-6), oleoylethanolamide (OEA) or linoleoylethanolamide (LEA). Data shown are the mean \pm SEM of three independent experiments . Results are expressed as the fold increase over ethanol control of normalised firefly luciferase activity. Asterisks represent statistically significant differences to activities in ethanol controls $(*, P<0.01)$.

fenofibrate and clofibric acid) were tested with plaice PPARs at concentrations of 10 and 100 $\mu \mathrm{M}$. When added to Gal4-PPAR $\alpha$ transfected cells both gemfibrozil and ciprofibrate, at concentrations of $100 \mu \mathrm{M}$, were able to increase transcriptional activity of this receptor, increasing activity 58-fold and 5.5-fold, respectively (Figure 3). Bezafibrate had no effect on Gal4-PPAR $\alpha$ activity from plaice. However, when added to Gal4-PPAR $\beta$ transfected cells at concentrations of $10 \mu \mathrm{M}$ and $100 \mu \mathrm{M}$, bezafibrate was able to induce a 2.3 -fold and 48 -fold induction in transcriptional activation respectively (Figure 3). None of the fibrates tested were able to induce the transcriptional activity of Gal4-PPAR $\gamma$ from plaice (data not shown).

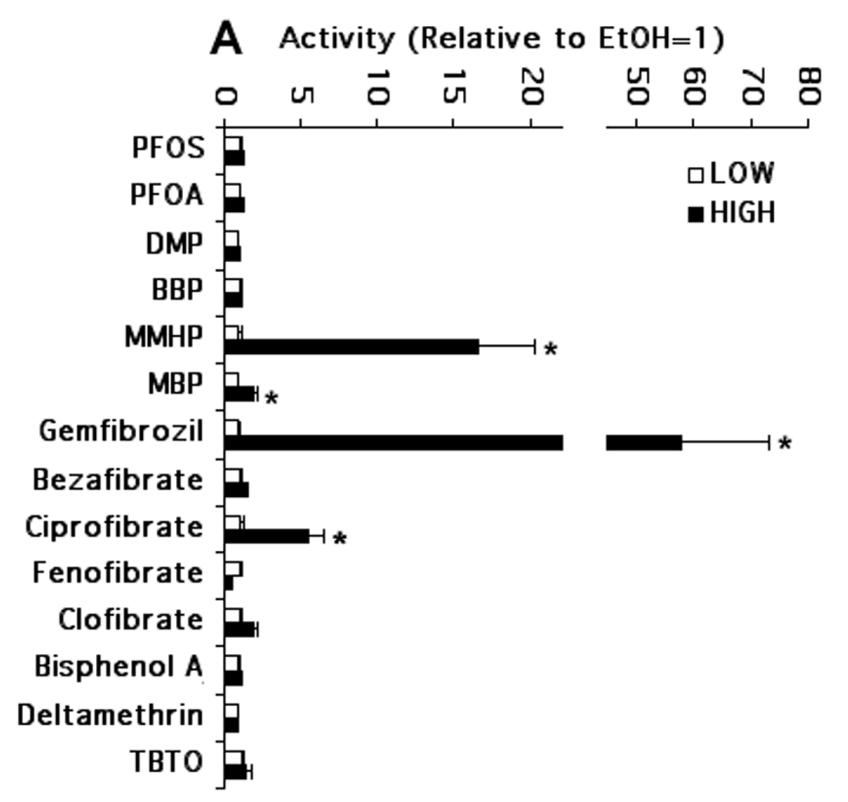

B Activity (Relative to $\mathrm{EtOH}=1$ )

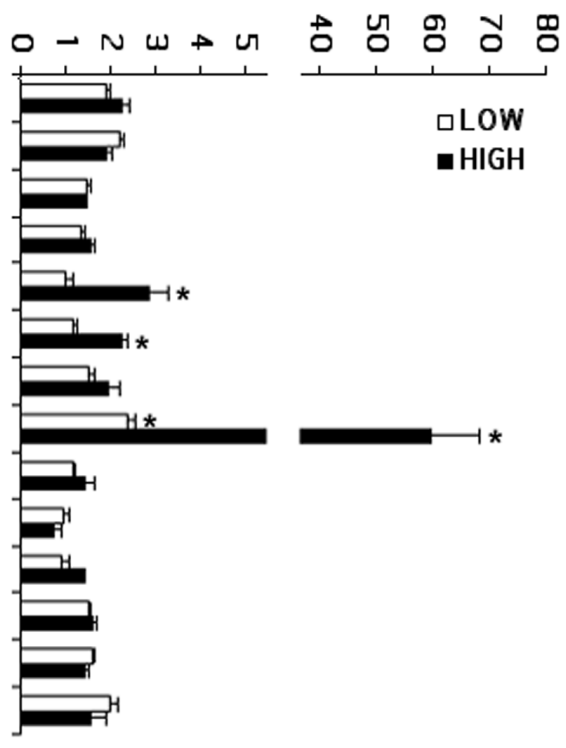

Figure 3. Activation of PPAR $\alpha$ or PPAR $\beta$ by aquatic contaminants.

FHM cells were transfected and treated as described in the legend of Figure 1. A, effects on PPAR $\alpha$; B, effects on PPAR $\beta$. Cells were treated with two concentrations per contaminant : Fluorooctanesulfonic acid (PFOS), perfluorooctanoic acid (PFOA),

dimethylphthalate (DMP), benzylbutylphthalate (BBP), bisphenol A and deltamethrin were tested at $10 \mu \mathrm{M}(\mathrm{LOW})$ and $25 \mu \mathrm{M}$ (HIGH). Mono-1-methylhexylphthalate (MMHP), 1-monobenzylbutylphthalate (MBP), gemfibrozil, bezafibrate, ciprofibrate, fenofibrate and clofibric acid were tested at $10 \mu \mathrm{M}$ (LOW) and $100 \mu \mathrm{M}$ (HIGH). Bis(tributyltin)oxide (TBTO) was tested at $10 \mathrm{nM}$ (LOW) and $50 \mathrm{nM}(\mathrm{HIGH})$. Data are the means \pm SEM of three independent experiments. Results are expressed as the fold increase over ethanol control of normalised firefly luciferase activity. Asterisks represent statistically significant differences to activities in ethanol controls ( $\left.{ }^{*}, P<0.01\right)$. 
The phthalate plasticizers, dimethylphthalate and benzylbutylphthalate, along with their mono-ester equivalents, mono-1-methylhexylphthalate and 1-monobenzylphthalate, were also tested for their ability to activate piscine PPARs. Neither of the parent phthalate compounds was able to activate any of the Gal4-PPARs. In contrast, both mono-1-methylhexyl phthalate and 1monobenzyl phthlate were able to significantly induce the transcriptional activities of Gal4-PPAR $\alpha$ and Gal4-PPAR $\beta$ at $100 \mu \mathrm{M}$. Compared to treatment with an ethanol vehicle, inductions in activity by mono-1-methylhexyl phthalate for Gal4-PPAR $\alpha$ and Gal4-PPAR $\beta$ were 16-fold and 2-fold, respectively. 1-monobenzyl phthalate induced both Gal4-PPAR $\alpha$ and Gal4-PPAR $\beta$ activity about 2fold at $100 \mu \mathrm{M}$ but had no effect at $10 \mu \mathrm{M}$.

Fluorooctanesulfonic acid (PFOS) and perfluorooctanoic (PFOA), used in the manufacture of fluoropolymers, and bisphenol A, used in the plastics industry had no effect with any construct. Also, the pyrethroid insecticide, deltamethrin, and the antifoulant, TBTO, failed to affect luciferase activity in the presence of any of the plaice PPAR constructs.

\subsection{TBTO is a potent inhibitor of plaice PPAR $\alpha$ and PPAR $\beta$}

In addition to testing pollutants for agonist activity on fish PPARs, compounds were also tested for their ability to inhibit the response of Gal4-PPAR $\alpha$ to Wy14643 and that of Gal4-PPAR $\beta$ to GW501506 (Figure 4). No potent activator of fish PPAR $\gamma$ has been identified and so PPAR $\gamma$ was omitted from antagonism studies. PFOS, PFOA, dimethylphthalate, benzylbutylphtalate, gemfibrozil, bisphenpolA and deltramethrin did not significantly affect induction of Gal4-PPAR $\alpha$ or Gal4-PPAR $\beta$ transactivation activity by model inducers. However relatively low concentrations of TBTO (50nM) antagonised Gal4-PPAR $\alpha$ activation.

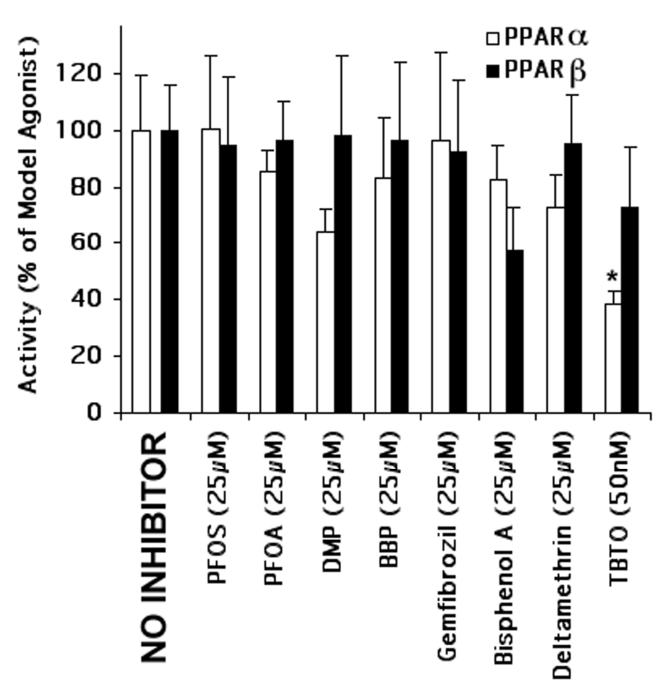

Fig. 4. Antagonism of PPAR $\alpha$ and PPARß by aquatic contaminants.

Transfected FHM cells were treated with Wy14643 and GW501516 (model activators), respectively, alone or in combination with an aquatic contaminant (see the legend of Figure 1 for experimental details). Means \pm SEM of three independent experiments are shown. Data are expressed as the percentage of normalised firefly luciferase luminescence, with respect to treatment with Wy14643 $(25 \mu \mathrm{M})$ and GW501516 $(10 \mu \mathrm{M})$ alone (NO INHIBITOR). Asterisks represent statistically significant differences to the treatment without inhibitor $(*, P<0.05)$. 
The effects of TBTO were further assessed over a concentration range (Figure 5). Inhibitory effects of TBTO on the stimulation of Gal4-PPAR $\alpha$ activity by WY14643 were detectable from the lowest concentration tested $(1 \mathrm{nM})$. Moreover, in this experiment the highest concentration of TBTO $(50 \mathrm{nM})$ partially inhibited the activation of Gal4-PPAR $\beta$ activity by GW501506 (Figure 5). In range finding tests, TBTO had shown cell toxic effects at $500 \mathrm{nM}$ (data not shown). To exclude the possibility that the apparent antagonistic effects of TBTO on PPAR transactivation were due to nonspecific effects on transcription rather than a specific interaction with PPARs, we tested the effect of TBTO on luciferase activities observed in FHM cells transfected with a positive control two hybrid construct system ( pBIND ID and pACTmyoD, Promega), which expresses a Gal4-Id fusion protein which interacts with an expressed myoD domain fused to a herpes virus VP16 transactivation domain. With this system TBTO did not affect luciferase expression (Figure 5)

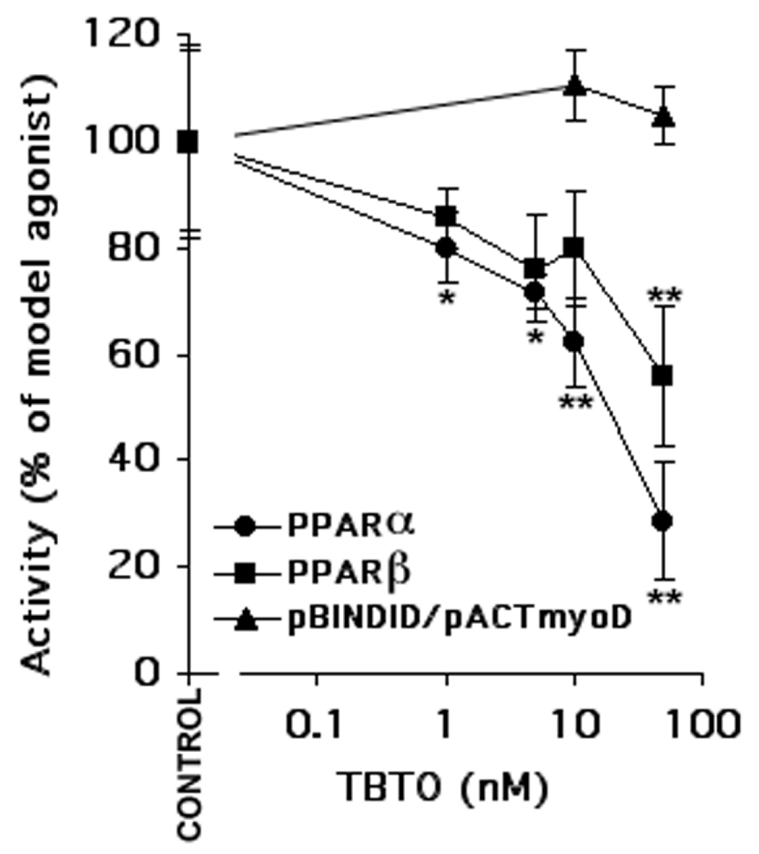

Fig. 5. Concentration dependent effects of TBTO on plaice PPAR $\alpha$ and PPARß.

FHM cells transfected with either Gal4-PPAR $\alpha$ and Gal4PPAR $\beta$ were treated with a model ligand (Wy14643 or GW501516, respectively) in combination with various concentrations of TBTO (see the legend of figure 1 for experimental details). Means \pm SEM of three independent experiments are shown. Data are expressed as the percentage of normalised firefly luciferase luminescence observed in CONTROL treatments with Wy14643 and GW501516 alone. Asterisks represent statistically significant differences to treatment with model ligand alone at $P<0.05$ (*) or $P<0.01$ $(* *)$. As an external control, cells transfected with pBIND ID and pACTmyoD, which results in constitutive luciferase expression, were included to test for general effects of TBTO on gene expression.

\section{Discussion}

Assessing the effects of putative ligands or of chemical pollutants on native PPARs is complicated by the fact that PPARs are active as heterodimers with RXRs, and RXR ligands also activate PPAR-RXR heterodimers (Mukherjee et al., 1998). To enable the study of chemical effects on PPAR in the absence of interaction effects with RXR-ligands, we isolated flounder PPAR ligandbinding domains and fused them to a yeast Gal4 DNA-binding domain. Co-transfection of fish cells with cDNAs encoding these fusion proteins together with a luciferase reporter gene driven by a 
promoter containing Gal4 binding sites enabled the measurement of PPAR activation in the absence of RXR, with reporter gene expression presumably being mediated by endogenous PPARinteracting cellular co-activator proteins. Using this system it was possible to demonstrate strong PPAR $\alpha$-driven reporter gene expression in the presence of Wy14643, previously shown to activate both mammalian and piscine PPAR $\alpha /$ RXR heterodimers. The mammalian PPAR $\beta$-specific ligand GW501516 activated both flounder PPAR $\alpha$ and PPAR $\beta$, but maximally only at concentrations considerably higher than those activating mammalian PPAR $\beta$. GW501506 has an $\mathrm{EC}_{50}$ of $1 \mathrm{~nm}$ with human PPAR $\beta$ and greater than $1 \mu \mathrm{M}$ for PPAR $\alpha$ (Oliver et al., 2001), whereas approximate EC50s for plaice PPAR $\beta$ and PPAR $\alpha$ are $1 \mu \mathrm{M}$ and $4 \mu \mathrm{M}$ respectively (data not shown).

Fatty acids and their derivatives also activated both flounder PPAR $\alpha$ and PPAR $\beta$, and at concentrations similar to those reported in mammals (Göttlicher et al., 1992;Issemann et al., 1993). The effects of selected fatty acids were also tested over a range of concentrations. In general for most fatty acids and for both Gal4-PPAR $\alpha$ and Gal4-PPAR $\beta$, luciferase activity was not increased below a fatty acid concentration of $60 \mu \mathrm{M}$, with the exception of $\gamma$-linoleic acid which was active with Gal4-PPAR $\beta$ at concentrations above $30 \mu \mathrm{M}$ (data not shown). In line with mammals, a number of fatty acids induced transactivation of plaice GAL4-PPAR $\alpha$ in this study. The most active fatty acid-like compounds were OEA and LEA.OEA is also a potent agonist of mammalian PPAR $\alpha$, and appears to have roles in controlling feeding through PPAR interactions (Schwartz et al., 2008). Notably in our study there was a tendency for fatty acids themselves to induce greater activity as the degree of unsaturation increased from 16:1 up to 20:5n-3 (eicosapentenoic acid). Docosahexaenoic acid (22:6n-3; DHA) was less effective. A previous study on native plaice PPARs indicated that DHA activated PPAR $\gamma$ in preference to PPAR $\alpha$ and PPAR $\beta$ (Leaver et al., 2005). However, neither plaice Gal4-PPAR $\gamma$ nor Gal4-PPAR $\beta$ was affected by DHA. DHA has been reported to activate mouse RXR (de Urquiza et al., 2000), indicating that in the previous study on fish, DHA may have been acting on RXR, the endogenous heterodimeric partner for transfected PPAR. Plaice PPAR $\gamma$, like PPAR $\gamma$ of other fish species, differs in structure from mammalian PPAR $\gamma$ and from all PPAR $\alpha$ and PPAR $\beta$ proteins at amino acid residues critical for ligand binding (Leaver et al., 2005;Maglich et al., 2003). Importantly, from previous studies and from the experiments reported here, it is apparent that fish PPAR $\gamma$ does not have high levels of constitutive activity. In the Gal4 assay, reporter gene activity driven by plaice PPAR $\gamma$ was very low and at background levels. The low constitutive activity of piscine PPAR $\gamma$, the high degree of conservation amongst fish species and the lack of response to fatty acids or mammalian subtype specific ligands suggests that if endogenous and other ligands for piscine PPAR $\gamma$ exist, they are likely to have some significant structural differences compared to fatty acids. 
Several environmental pollutants, some of which have been reported to activate mammalian PPARs, were also tested with the plaice Gal4-PPAR chimerae. Amongst the compounds tested were several of the fibrate drugs, developed as subtype specific agonists of human PPAR $\alpha$. The presence of these fibrate drugs has been reported in sewage treatment effluent and consequently they ultimately accumulate in the aquatic environment (Buser and Müller, 1998;Ternes, 1998;Andreozzi et al., 2003;Sanderson et al., 2003;Fent et al., 2006). Thus there is some interest in their possible effects on fish living in contaminated environments. In humans and rodents clofibrate, gemfibrozil and ciprofibrate are an order of magnitude more potent on PPAR $\alpha$ than PPAR $\beta$ or PPAR $\gamma$, whilst bezafibrate is an almost equally effective agonist of all three subtypes (Willson et al., 2000; Mukherjee et al., 2002). Plaice differ in that bezafibrate is selective for PPAR $\beta$ which is similar to the situation in the African clawed frog (Xenopus laevis), where bezafibrate activates PPAR $\beta$ but has little or no effect on PPAR $\alpha$ or PPAR $\gamma$ (Krey et al., 1997). These previous data and the results here suggest fibrates to be targets of PPARs in a diverse range of species including mammals, fish and amphibians. Our results indicate that at least some of the fibrate drugs present in the aquatic environment could exert toxic effects in fish via PPAR mediated pathways, but that the concentrations required $(>10 \mu \mathrm{M})$ far exceed measured environmental concentrations of individual fibrates, maximum concentrations of which have been reported between $0.02 \mu \mathrm{M}$ and $0.2 \mu \mathrm{M}$ in various sewage treatment effluents (Buser and Müller, 1998;Ternes, 1998;Andreozzi et al., 2003; Sanderson et al., 2003; Kolpin et al., 2002;Stumpf et al., 1999). However, the demonstration that multiple fibrates can activate fish PPARs shows that additive effects in the environment should be considered.

Phthalates are compounds used in the manufacturing industry as plasticizers, which serve to soften PVC products. In addition they are often found in cosmetics, perfumes, industrial paints and solvents and certain drugs, and measured environmental concentrations can be similar to concentrations causing laboratory effects in some animals (Oehlmann et al., 2009). Phthalates also cause peroxisome proliferation when given to laboratory rodents and are ligands for mammalian PPARs, and have consequently been linked to rising levels of obesity and diabetes in exposed humans (Desvergne et al., 2009). Both benzylbutylyphthalate and dimethylphthalate failed to increase PPAR-driven reporter gene expression at concentrations up to $25 \mu \mathrm{M}$. However, due to the biotransformation process that occurs within organisms, parent phthalate compounds are metabolized to their monophthalate esters and therefore two of these transformation products were selected for testing in our in vitro assay system. It has previously been reported that mono-1methyl-hexyl-pthalate can activate both murine and human PPAR $\alpha$ and PPAR $\gamma$ at concentrations above $62.5 \mu \mathrm{M}$ (Lampen et al., 2003). In agreement with these findings mono-1-methyl-hexylphthalate proved to be an effective, but not particularly potent, activator of plaice PPAR $\alpha$ and also 
of PPAR $\beta$. In contrast to mammalian PPAR $\gamma$, mono-1-methyl-hexyl-phthalate had no effect on PPAR $\gamma$-dependent reporter gene expression. At $100 \mu \mathrm{M}$, mono-benzyl-phthalate activated plaice PPAR $\alpha$ and PPAR $\beta$ isotypes, although only slightly (2-fold). In rodents, mono-benzyl-phthalate is an activator of all three PPAR subtypes (Lampen et al., 2003), although with apparently more potent effects on PPAR $\alpha$ and PPAR $\gamma$ than PPAR $\beta$.

In previous studies on fish PPARs, PFOA has been shown to transactivate PPAR/RXR heterodimers (Leaver et al., 2005) and in mice and humans both PFOA and PFOA act as weak agonists in a similar Gal4-PPAR assay to that used here (Takacs and Abbott, 2007). However, plaice Gal4-PPAR was not affected by PFOA or PFOS, which suggests that, as explained above for DHA, these compounds may be targeting RXR in fish. Even in mice the effects of PFOA on PPAR-target genes appear to be to some extent independent of PPAR $\alpha$ (Rosen et al., 2008), indicating that other mechanisms of effect should be investigated.

We also tested environmental pollutants for inhibition of Wy14643 and GW501516-activated plaice PPAR $\alpha$ and PPAR $\beta$ respectively. Most significantly TBTO was found to be inhibitory to both plaice PPAR $\alpha$ and PPAR $\beta$ at concentrations as low as $1 \mathrm{nM}$ in cellular transfection assays. Recently TBTO and its analogs have been found to be potent nM agonists of mammalian and amphibian PPAR $\gamma$ and of mammalian and gastropod mollusc RXR (Nishikawa et al., 2004;Hiromori et al., 2009; Grün et al., 2006). The activation of RXR by TBT may underly the development of imposex in wild populations of molluscs (Nishikawa et al., 2004). In mice administration of TBTO at nM concentrations in utero causes subsequent increases in fat mass dependent on activation of PPAR $\gamma$ (Kirchner et al., 2010). There is no information regarding the effects of TBTO on fish RXR, but the potent inhibitory effects on PPAR $\alpha$ and PPAR $\beta$ and a lack of effect on PPAR $\gamma$ show that fish may have different responses to low level TBTO than mammals and amphibians. Nevertheless, these results suggest that fish inhabiting environments polluted with this compound could be metabolically impaired with respect to PPAR signalling. Measured concentrations of TBTO and related organotin compounds in the tissues of wild fish are often in the $\mu \mathrm{gKg}^{-1}$ range (Hoch, 2001; Antizar-Ladislao, 2008). For comparison, 10nM, a concentration at which plaice PPAR $\alpha$ was inhibited, is approximately $6 \mu \mathrm{gL}^{-1}$.

In conclusion plaice PPAR $\alpha$ and PPAR $\beta$ exhibit both similarities and differences to their mammalian counterparts. Some ligands such as Wy14643, GW501506 and some fibrates act as activators of plaice PPARs as they do in mammals, whilst other mammalian ligands are not effective in activating fish PPARs. Of the environmental pollutants tested, only TBTO had effects at environmentally relevant concentrations. Although environmental concentrations of TBT have been decreasing in many parts of the world following legislation to limit their use, they are still 
detectable in sediments and fish tissues in quantities (Antizar-Ladislao, 2008) that would, based on the results reported here, inhibit ex vivo piscine PPAR $\alpha$ and PPAR $\beta$. Given the potency of this contaminant and its persistent and ubiquitous presence in estuarine environments, these effects should be further studied in vivo, to establish what, if any, are the physiological consequences and knock-on ecological effects of exposure.

Acknowledgements: Louise Colliar was supported by a UK Natural Environment Research Council (NERC) studentship, NE/F008287/1.

\section{References}

Andreozzi,R., Raffaele,M. and Nicklas,P., 2003. Pharmaceuticals in STP effluents and their solar photodegradation in aquatic environment. Chemosphere. 50, 1319-1330.

Antizar-Ladislao, B., 2008. Environmental levels, toxicity and human exposure to tributyltin (TBT)-contaminated marine environment. A review. Environment International 34, 292 308.

Bility,M.T., Thompson,J.T., Mckee,R.H., David,R.M., Butala,J.H., Vanden Heuvel,J.P. and Peters,J.M., 2004. Activation of mouse and human peroxisome proliferator-activated receptors (PPARs) by phthalate monoesters. Toxicological Sciences. 82, 170-182.

Buser,H. and Müller,M.D., 1998. Occurrence of the pharmaceutical drug clofibric acid and the herbicide mecoprop in various Swiss lakes and in the North sea. Environmental Science and Technology. 32, 188-192.

de Urquiza,A.M., Liu,S., Sjöberg,M., Zetterström,R.H., Griffiths,W., Sjövall,J. and Perlmann,T., 2000. Docosahexaenoic acid, a ligand for the retinoid X receptor in mouse brain. Science. 290, 2140-2144.

Desvergne,B., Michalik,L. and Wahli,W., 2006. Transcriptional regulation of metabolism. Physiological Reviews. 86, 465-514.

Desvergne,B., Feige,J.N. and Casals-Casas,C., 2009. PPAR-mediated activity of phthalates: A link to the obesity epidemic? Molecular and Cellular Endocrinology. 304, 43-48.

Dyer,B.W., Ferrer,F.A., Klinedinst,D.K. and Rodriguez,R., 2000. A noncommercial dual luciferase enzyme assay system for reporter gene analysis. Analytical Biochemistry 282, 161-164.

Escher, P., Braissant, O., Basu-Modak, S., Michalik, L., Wahli, W., Desvergne B. 2001. Rat PPARs; quantitative analysis in adult rat tissues and regulation in fasting and refeeding.

Endocrinology 142, 4195-4202. 
Feige,J.N., Gelman,L., Michalik,L., Desvergne,B. and Wahli,W., 2006. From molecular action to physiological outputs: Peroxisome proliferator-activated receptors are nuclear receptors at the crossroads of key cellular functions. Progress in Lipid Research. 45, 120-159.

Fent,K., Weston,A.A. and Caminada,D., 2006. Ecotoxicology of human pharmaceuticals. Aquatic Toxicology. 76, 122-159.

Fievet,C. and Staels,B., 2009. Efficacy of peroxisome proliferator-activated receptor agonists in diabetes and coronary artery disease. Current Atherosclerosis Reports. 11, 281-288.

Göttlicher,M., Widmark,E., Li,Q. and Gustafsson,J., 1992. Fatty acids activate a chimera of the clofibric acid-activated receptor and the glucocorticoid receptor. Proceedings of the National Academy of Sciences of the United States of America. 89, 4653-4657.

Grün,F. and Blumberg,B., 2009. Minireview: The Case for Obesogens. Molecular Endocrinology. $23,1127-1134$.

Grün,F., Watanabe,H., Zamanian,Z., Maeda,L., Arima,K., Cubacha,R., Gardiner,D.M., Kanno,J., Iguchi,T. and Blumberg,B., 2006. Endocrine-disrupting organotin compounds are potent inducers of adipogenesis in vertebrates. Molecular Endocrinology. 20, 2141-2155.

Hihi,A.K., Michalik,L. and Wahli,W., 2002. PPARs: Transcriptional effectors of fatty acids and their derivatives. Cellular and Molecular Life Sciences. 59, 790-798.

Hiromori,Y., Nishikawa,J., Yoshida,I., Nagase,H. and Nakanishi,T., 2009. Structure-dependent activation of peroxisome proliferator-activated receptor (PPAR) $\gamma$ by organotin compounds. Chemico-Biological Interactions. 180, 238-244.

Hoch,M., 2001. Organotin compounds in the environment - an overview. Applied Geochemistry. 16, 719-743.

Issemann,I., Prince,R.A., Tugwood,J.D. and Green,S., 1993. The peroxisome proliferator-activated receptor:retinoid $\mathrm{X}$ receptor heterodimer is activated by fatty acids and fibrate hypolipidaemic drugs. Journal of Molecular Endocrinology. 11, 37-47.

Kersten, S., Seydoux, J., Peters, J.M., Gonzalez, F.J., Desvergne, B., Wahli, W. 1999. Peroxisome proliferator-activated receptor $\alpha$ mediates the adaptive response to fasting. Journal of Clinical Investigation 103, 1489 -1498.

Kirchner,S., Kieu,T., Chow,C., Casey,S. and Blumberg,B., 2010. Prenatal Exposure to the Environmental Obesogen Tributyltin Predisposes Multipotent Stem Cells to Become Adipocytes. Molecular Endocrinology. 24, 526-539. 
Kolpin,D.W., Furlong,E.T., Meyer,M.T., Thurman,E.M., Zaugg,S.D., Barber,L.B. and Buxton,H.T., 2002. Pharmaceuticals, hormones, and other organic wastewater contaminants in US streams, 1999-2000: A national reconnaissance. Environmental Science \& Technology. 36, 1202-1211.

Krey,G., Braissant,O., L'Horset,F., Kalkhoven,E., Perroud,M., Parker,M.G. and Wahli,W., 1997. Fatty acids, eicosanoids, and hypolipidemic agents identified as ligands of peroxisome proliferator-activated receptors by coactivator-dependent receptor ligand assay. Molecular Endocrinology. 11, 779-791.

Lampen,A., Zimnik,S. and Nau,H., 2003. Teratogenic phthalate esters and metabolites activate the nuclear receptors PPARs and induce differentiation of F9 cells. Toxicology and Applied Pharmacology. 188, 14-23.

Leaver,M.J., Boukouvala,E., Antonopoulou,E., Diez,A., Favre-Krey,L., Ezaz,M.T., Bautista,J.M., Tocher,D.R. and Krey,G., 2005. Three peroxisome proliferator-activated receptor isotypes from each of two species of marine fish. Endocrinology. 146, 3150-3162.

Leaver,M.J., Ezaz,M.T., Fontagne,S., Tocher,D.R., Boukouvala,E. and Krey,G., 2007. Multiple peroxisome proliferator-activated receptor $\beta$ subtypes from Atlantic salmon (Salmo salar). Journal of Molecular Endocrinology. 38, 391-400.

Leone, T.C, Weinheimer, C.J., Kelly, D.P. 1999. A critical role for the peroxisome proliferatorsactivated receptor $\alpha(\mathrm{PPAR} \alpha)$ in the cellular fasting response: The PPAR $\alpha$-null mouse as a model of fatty acid oxidation disorders. Proceedings of the National Acadamy of Sciences USA $96,7473-7478$.

Maglich,J.M., Caravella,J.A., Lambert,M.H. and Willson,T.M., 2003. The first completed genome sequence from a teleost fish (Fugu rubripes) adds significant diversity to the nuclear receptor superfamily. Nucleic Acids Research. 31, 4051-4058.

Mukherjee,R., Strasser,J., Jow,L., Hoener,P., Paterniti,J.R. and Heyman,R.A., 1998. RXR agonists activate PPAR $\alpha$-inducible genes, lower triglycerides, and raise HDL levels in vivo. Arteriosclerosis, Thrombosis and Vascular Biology. 18, 272-276.

Mukherjee,R., Sun,S.X., Santomenna,L., Miao,B., Walton,H., Liao,B., Locke,K., Zhang,J.H., Nguyen,S.H., Zhang,L.T., Murphy,K., Ross,H.O., Xia,M.X., Teleha,C., Chen,S.Y., Selling,B., Wynn,R., Burn,T. and Young,P.R., 2002. Ligand and coactivator recruitment preferences of peroxisome proliferator activated receptor alpha. Journal of Steroid Biochemistry and Molecular Biology. 81, 217-225.

Nishikawa,J., Mamiya,S., Kanayama,T., Nishikawa,T., Shiraishi,F. and Horiguchi,T., 2004. Involvement of the retinoid $\mathrm{X}$ receptor in the development of imposex caused by organotins in gastropods. Environmental Science \& Technology. 38, 6271-6276. 
Oehlmann,J., Schulte-Oehlmann,U., Kloas,W., Jagnytsch,O., Lutz,I., Kusk,K.O., Wollenberger,L., Santos,E.M., Paull,G.C., Van Look,K.J.W. and Tyler,C.R., 2009. A critical analysis of the biological impacts of plasticizers on wildlife. Philosophical Transactions of the Royal Society B-Biological Sciences. 364, 2047-2062.

Oliver,W.R., Shenk,J.L., Snaith,M.R., Russell,C.S., Plunket,K.D., Bodkin,N.L., Lewis,M.C., Winegar,D.A., Sznaidman,M.L., Lambert,M.H., Xu,H.E., Sternbach,D.D., Kliewer,S.A., Hansen,B.C. and Willson,T.M., 2001. A selective peroxisome proliferator-activated receptor delta agonist promotes reverse cholesterol transport. Proceedings of the National Academy of Sciences of the United States of America. 98, 5306-5311.

Rosal,R., Rodea-Palomares,I., Boltes,K., Fernandez-Pinas,F., Leganes,F., Gonzalo,S. and Petre,A., 2010. Ecotoxicity assessment of lipid regulators in water and biologically treated wastewater using three aquatic organisms. Environmental Science and Pollution Research. $17,135-144$.

Rosen,M.B., Abbott,B.D., Wolf,D.C., Corton,J.C., Wood,C.R., Schmid,J.E., Das,K.P., Zehr,R.D., Blair,E.T. and Lau,C., 2008. Gene Profiling in the Livers of Wild-type and PPAR alpha-Null Mice Exposed to Perfluorooctanoic Acid. Toxicologic Pathology. 36, 592-607.

Rosen, E.D., Spiegelman, B.M. 2001. PPAR $\gamma$ : a nuclear regulator of metabolism, differentiation, and growth. Journal of Biological Chemistry 276. 37731 -37734.

Sanderson,H., Johnson,D.J., Wilson,C.J., Brian,R.A. and Solomon,K.R., 2003. Probablistic hazard assessment of enviornmentally occurring pharmaceuticals toxicity to fish, daphnids and algae by ECOSAR screening. Toxicology Letters. 144, 395.

Schwartz,G.J., Fu,J., Astarita,G., Li,X., Gaetani,S., Campolongo,P., Cuomo,V. and Piomelli,D., 2008. The lipid messenger OEA links dietary fat intake to satiety. Cell Metabolism. 8, 281288.

Stumpf,M., Ternes,T.A., Wilken,R.D., Rodrigues,S.V. and Baumann,W., 1999. Polar drug residues in sewage and natural waters in the state of Rio de Janeiro, Brazil. Science of the Total Environment. 225, 135-141.

Takacs,M.L. and Abbott,B.D., 2007. Activation of mouse and human peroxisome proliferatoractivated receptors (alpha, beta/delta, gamma) by perfluorooctanoic acid and perfluorooctane sulfonate. Toxicological Sciences. 95, 108-117.

Ternes,T.A., 1998. Occurrence of drugs in German sewage treatment plants and rivers. Water Research. 32, 3245-3260.

Wagner K.D. and Wagner N., 2010. Peroxisome proliferator-activated receptor beta/delta $(\mathrm{PPAR} \beta / \delta)$ acts as regulator of metabolism linked to multiple cellular functions. Pharmacol Ther. 25, 423-435. 
Willson,T.M., Brown,P.J., Sternbach,D.D. and Henke,B.R., 2000. The PPARs: From orphan receptors to drug discovery. Journal of Medicinal Chemistry. 43, 527-550. 